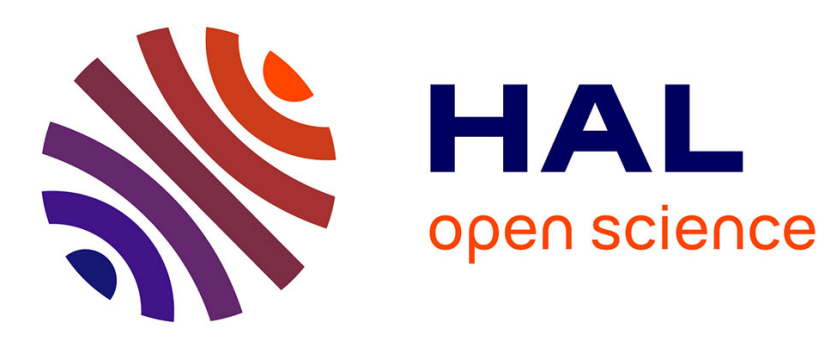

\title{
Foot ulcer prevention using biomechanical modelling
}

Vincent Luboz, Antoine Perrier, Ian Stavness, John Lloyd, Marek Bucki, Francis Cannard, Bruno Diot, Nicolas Vuillerme, Yohan Payan

\section{To cite this version:}

Vincent Luboz, Antoine Perrier, Ian Stavness, John Lloyd, Marek Bucki, et al.. Foot ulcer prevention using biomechanical modelling. Computer Methods in Biomechanics and Biomedical Engineering: Imaging \& Visualization, 2014, 2 (4), pp.189 - 196. 10.1080/21681163.2013.837410 . hal-01092588

\section{HAL Id: hal-01092588 \\ https://hal.science/hal-01092588}

Submitted on 9 Dec 2014

HAL is a multi-disciplinary open access archive for the deposit and dissemination of scientific research documents, whether they are published or not. The documents may come from teaching and research institutions in France or abroad, or from public or private research centers.
L'archive ouverte pluridisciplinaire HAL, est destinée au dépôt et à la diffusion de documents scientifiques de niveau recherche, publiés ou non, émanant des établissements d'enseignement et de recherche français ou étrangers, des laboratoires publics ou privés. 


\title{
Foot Ulcer Prevention Using Biomechanical Modeling
}

\author{
V. Luboz \\ A. Perrier \\ I. Stavness, J. E. Lloyd \\ UJF-Grenoble 1/CNRS/TIMC- Laboratoires TIMC-IMAG et University of British Columbia \\ IMAG UMR 5525 38041, La \\ AGIM, La Tronche, France \\ Vancouver, Canada \\ Tronche, France \\ vluboz@imag.fr \\ TexiSense, Montceau-les- \\ stavnessegmail.com \\ lloydecs.ubc.ca \\ M. Bucki, F. Cannard \\ TexiSense, Montceau-les- \\ Mines, France \\ Mines, France \\ marek.bucki@texisens \\ antoine.perrier@imag \\ e.com \\ francis.cannardetexi \\ fr \\ B. Diot \\ N. Vuillerme \\ sense.com \\ Y. Payan \\ Laboratoire AGIM, La Tronche, France Laboratoire AGIM, La Tronche, France \\ IDS, Montceau-les-Mines, France \\ \& Institut Universitaire de France \\ b.dioteids-assistance.com \\ Nicolas.Vuillermedagim.eu \\ UJF-Grenoble 1/CNRS/TIMC-IMAG \\ UMR 5525 38041La Tronche, France \\ ypayaneimag.fr
}

\begin{abstract}
Foot ulcers are a common complication of diabetes and are the consequence of trauma to the feet and a reduced ability to perceive pain in persons with diabetes. Ulcers appear internally when pressures applied on the foot create high internal strains below bony structures. It is therefore important to monitor tissue strains in persons with diabetes. We propose to use a biomechanical model of the foot coupled with a pressure sensor to estimate the strains within the foot and to determine if they can cause ulcer formation. Our biomechanical foot model is composed of a Finite Element mesh representing the soft tissues, separated into four Neo Hookean materials with different elasticity: plantar skin, non-plantar skin, fat and muscles. Rigid body models of the bones are integrated within the mesh to rigidify the foot. Thirty-three joints connect those bones around cylindrical or spherical pivots. Cables are included to represent the main ligaments in order to stabilize the foot. This model simulates a realistic behavior when the sole is subjected to pressures measured with a sensor during bipedal standing. Surface strains around $5 \%$ are measured below the heel and metatarsal heads while internal strains are close to $70 \%$. This strain estimation, when coupled to a pressure sensor, could consequently be used in a patient alert system to prevent ulcer formation.
\end{abstract}

Keywords: foot ulcer prevention, biomechanical model.

\section{Introduction}

Diabetes affects the lower limb through long-term chronic complications, including peripheral neuropathy and angiopathy [1]. When the diabetic foot is subject to a trauma, such as repetitive stress from high pressure, illfitting footwear rubbing on the skin or an object inside the shoe, an ulcer can form. This condition if often followed by amputation of a toe or foot: a limb is lost every 30 seconds on Earth because of diabetes. In 2007, in the US, the cost of ulcers was 48 billions USD [2] and life expectancy after foot amputation was $50 \%$ after 5 years [3].

The two main factors that promote ulcer development are the excessive intensity and the repetition of pressures on the foot. This is worsened by diabetic neuropathy which reduces or even suppresses sensation in the feet of persons with diabetes. Three mechanisms are linked to the pressures leading to ulcer [4]: (1) ischemia caused by increased pressure duration, (2) large tissue strains created by increased pressure magnitude, or (3) tissue fatigue caused by increased number of pressure loads.

Today, prevention of foot ulcers is mainly based on the patient's daily vigilance and on frequent medical doctor monitoring. This approach is consequently dependent on the patient's involvement which tends to decrease when no external signs are visible. Unfortunately, when the first ulcers appear, serious complications have already begun for the diabetic patient because of diabetic angiopathy, which limits tissue healing and increases recurrence. Reducing the pressure load at the interface between the diabetic foot and the trauma source prevents further ulceration and facilitates wound healing [5]. Pressure loads can be reduced with devices such as casts, orthotics, insoles or foam bandages.

To aid patient monitoring, devices measuring pressures at the foot/insole interface were introduced few years ago [6][7][8]. Novel (http://www.novel.de), Tekscan (http://www.tekscan.com), Vista Medical (http://www.pressuremapping.com) and Orpyx (http://orpyx.com/) now propose commercial devices that are composed of few pressure sensors located under the heel and the metatarsal heads. Unfortunately, their price and their need to be constantly connected to monitor the plantar pressures in real time make them difficult to use in a daily long-term prevention routine. Moreover, these devices are limited to measuring pressures at the skin surface and it is well known that deep pressure ulcers stem from internal stresses and strains. Such ulcers usually start 
in deep tissues and progress outward rapidly, causing substantial subcutaneous damage underneath intact skin. Deep pressure ulcers are therefore particularly dangerous since they may be difficult to detect visually. Devices that measure surface pressures are mainly used to alert persons suffering from diabetes to abnormal pressures that may cause skin damage [9]. These measurements, however, cannot predict ulcer formation due to internal tissue loading [10]. For example, a similar pressure distribution could be observed under the heel of a thin person with blunt calcaneus bone and a heavy diabetic person with sharp calcaneus bone; however, the likelihood of a pressure ulcer forming depends on the calcaneus bone curvature as well as the thickness of the soft tissues as proved in [11] for the ischial tuberosity.

In order to account for these anatomical differences and to quantitatively estimate the internal stresses and strains from the measured external pressures, several studies have proposed (1) to build a patient-specific biomechanical model of the foot including soft tissues and bony prominence, and (2) to use this numerical model to compute the internal strains and stresses [12]. Several Finite Element (FE) models have been introduced to simulate the foot deformations. Several studies such as [13] proposed 2D biomechanical models of the foot with fairly complex material behavior and anatomy, providing very interesting results in terms of stresses and strains within the foot. Nevertheless, the foot behavior is highly dependent on its whole 3D shape and the influence of each of its structures (bones or tendons for example). Also, it seems that clinicians prefer a 3D analysis of the foot to determine the development of a pressure ulcer. In [14], the foot soft tissues (skin, fat and muscles) are modeled as a 3D FE mesh with a homogeneous linear elastic material, the bones are modeled as another set of rigid FE meshes, while contacts between the bones simulate the joints, and cables connect the bones for the ligaments located in the mid foot. The 3D biomechanical model presented in [15] seems closer to reality: it models the soft tissues in a large deformations framework with a Mooney Rivlin constitutive law and it adds almost all the ligaments of the foot. However, the main drawback of these models is their long computation times: it can take hours to compute simple plantar loadings. For this reason, it would be difficult to integrate previously proposed foot models into a daily foot ulcer prevention process. In this article, we propose and discuss the challenges of defining a simple, yet realistic, biomechanical model that could estimate internal foot stresses and strains in interactive time.

\section{Materials and methods}

Our foot biomechanical model has been developed using the 3D simulation platform, ArtiSynth [16] (www.artisynth.org). The model is composed of soft tissues, bones, ligaments and joints.

\subsection{Soft tissue FE mesh}

The soft tissues are modeled as a FE mesh and are divided into four layers: the plantar skin (below the heel, the metatarsus, and the toes), the non-plantar skin, muscles and fat. The outer surface of this mesh was based on the skin surface from the Zygote database (www.zygote.com). Using an automatic FE mesh generator [17], the model skin surface was filled with finite elements. This generator aims at creating a mesh with a maximum of hexahedrons (to limit the locking effect observed for tetrahedral elements in quasi-incompressible assumptions) while keeping a controlled number of elements to enable fast FE computation. The accuracy of the surface fitting is made possible by optimally subdividing hexahedrons intersecting the organ surface into prisms, pyramids and/or tetrahedron depending on the geometry of the surface locally. Because we assume the bones to be rigid (see section 2.2), there is no need to represent them as FE meshes in our simulation. We therefore set the mesh generator to leave holes in the soft tissue mesh in order to represent the bones' geometry (also given by the Zygote database).

The FE mesh representing the foot soft tissues is shown in Figure 1 and is composed of 36,895 elements $(11,045$ hexahedrons, 10,206 pyramids, 9,991 tetrahedrons, and 5,653 wedges) and 22,774 nodes. Neo Hookean materials were chosen for the foot tissues in order to represent large deformations. The mesh has four layers, each with distinct material properties: Young moduli were set to $6 \mathrm{MPa}$ for the plantar skin, $200 \mathrm{kPa}$ for the rest of the skin, $50 \mathrm{kPa}$ for the muscles and $4 \mathrm{kPa}$ for the fat (Figure 2). Assuming these tissues are quasi-incompressible, we set their Poisson ratio to 0.495 . With the exception of the non-plantar skin, the values of the material properties were taken from measurements reported by Sopher [18] for an Ogden model and converted here to fit our Neo Hookean model (taking the equivalent Young initial modulus).

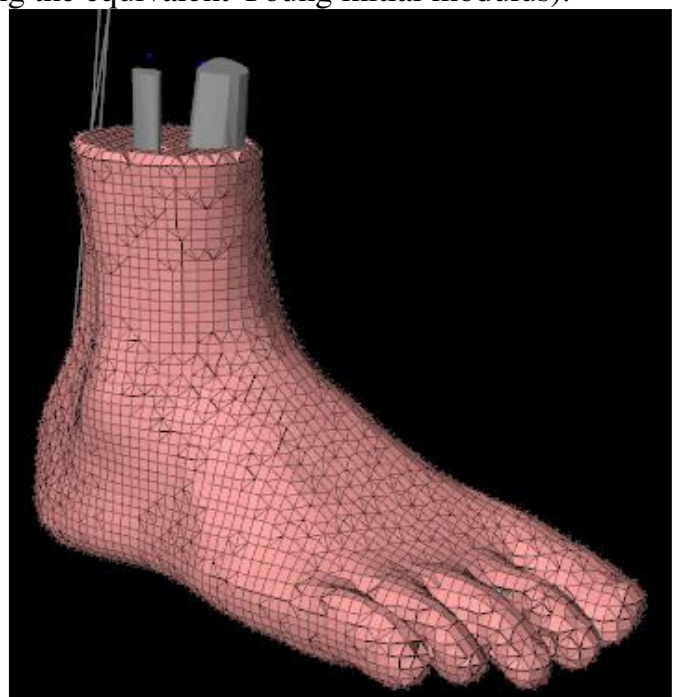


Figure 1. The surface of the FE mesh representing the foot soft tissues.

The most superficial layer of elements simulates the foot skin. Two different materials were chosen for the skin because of the high stiffness of the plantar skin compared to the rest of the skin. The elasticity modulus chosen for the non-plantar skin was measured using our own device: LASTIC (for Light Aspiration device for in vivo Soft TIssue Characterization). The LASTIC device is based on the pipette aspiration principle [19] and aims at characterizing in vivo the elastic modulus of soft tissues. It creates a negative pressure against the tissue surface on which it is placed and consequently deforms the surface of these tissues. The height of the deformation is measured on the images captured during the acquisition with several negative pressures. The corresponding height/negative pressure curve allows estimating the elastic modulus of the tissues through inverse method. LASTIC was used to estimate the elasticity of the foot arch skin of a healthy subject (Figure 3) and gave a value of $200 \mathrm{kPa}$. This value was used for the whole skin except for the plantar surface which was too stiff to be evaluated with LASTIC (as mentioned above, the $6 \mathrm{MPa}$ proposed by [18] was chosen for this plantar skin). To define the two skin layers, all the elements of the surface of the FE mesh that were below a certain height (in our case, about $2 \mathrm{~mm}$ ) in the model were considered as plantar skin (see Figure 2). The other surface elements were assigned the material with a Young modulus of $200 \mathrm{kPa}$ for the rest of the skin.

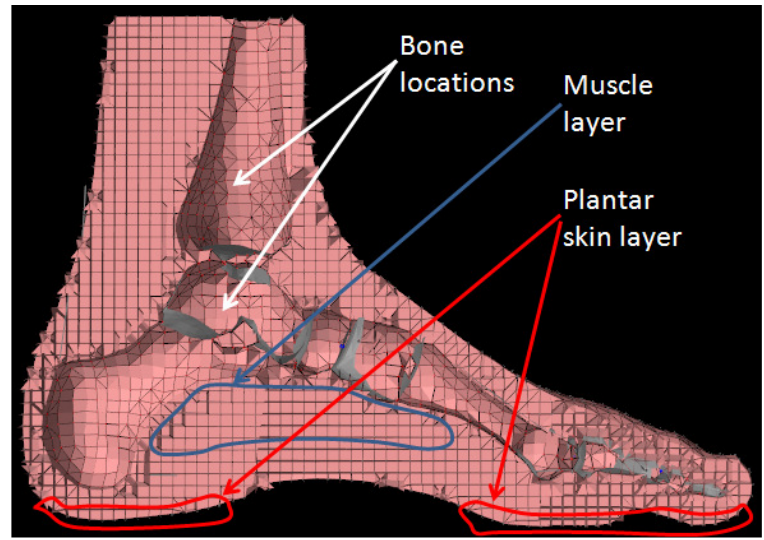

Figure 2. Cross section of the FE mesh representing the foot soft tissues. The plantar skin layer, the muscle layer and some gaps defining the bones' locations can be seen. The rest of the surface elements are part of the softer skin layer, while the other internal elements form the fat layer.

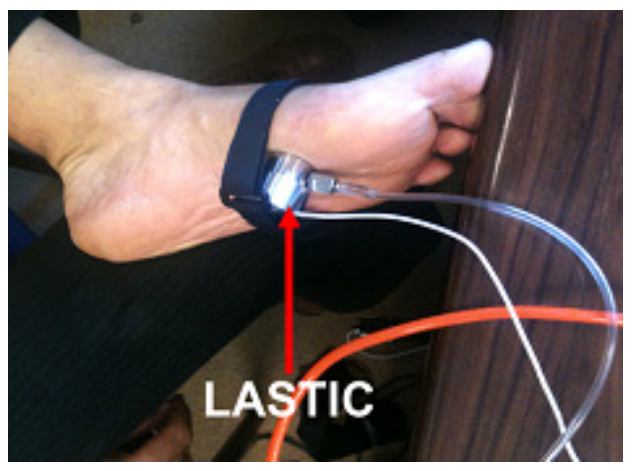

Figure 3. Acquisition of the elastic modulus of the skin below the foot arch using LASTIC.

The muscle layer was defined from the Zygote database and is limited for now to the muscles of the foot arch. This area was mapped in the FE mesh to find the elements considered as muscles (Figure 2). All other elements were assumed to be part of the fat layer.

\subsection{Bones and joints}

The ArtiSynth framework provides a very efficient computational formulation for the coupled simulation of rigid and deformable structures, with a constraint-based mechanism for attaching bones and soft tissues and the use of semi-implicit time integration [20]. The bones are therefore represented as rigid body surfaces in our model. The 26 foot bones and a section of the tibia and fibula are integrated in the model from the Zygote database (Figure 4). By not modeling the bones as FE meshes, we are able to decrease the FE matrix size and speed up the simulation.

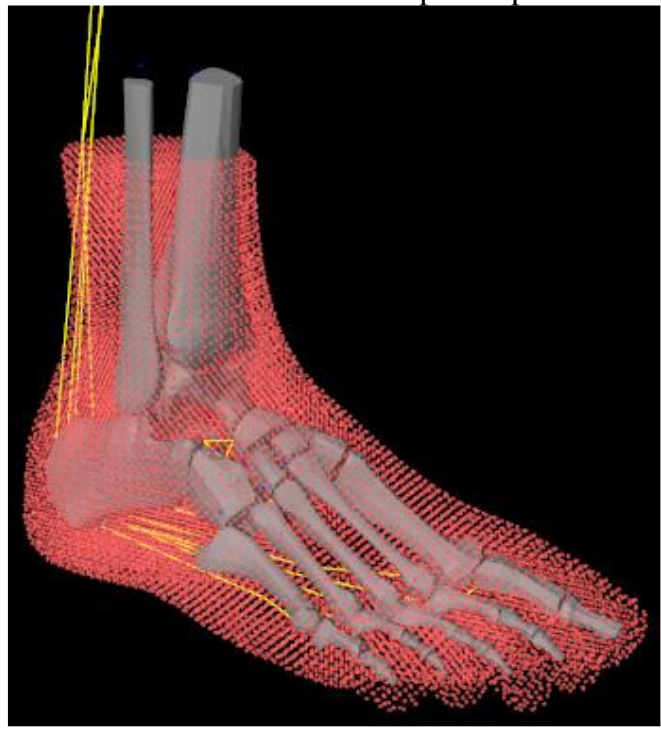

Figure 4. The bony structures within the foot model. Some tendons and ligaments are visible as yellow cables. 


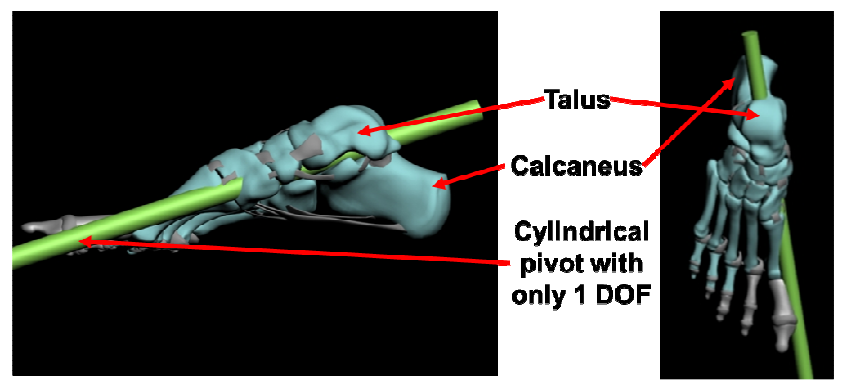

Figure 5. Cylindrical pivot (scaled up for visibility) representing the joint between the talus and calcaneus bones.

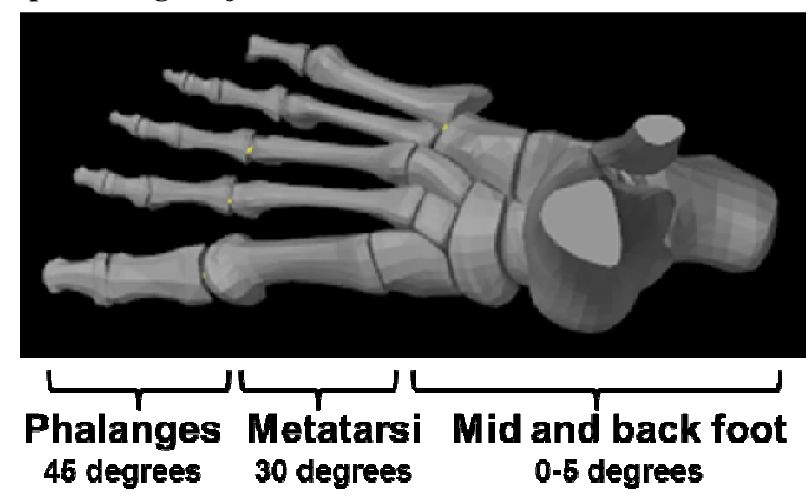

Figure 6. Pivots simulate the joints connecting the different bones. They allow different maximum angles depending on the foot regions: $\mathbf{4 5}$ degrees for the phalanges, $\mathbf{3 0}$ degrees for the metatarsi, and 0 to 5 degrees for the rest of the foot.

We worked with an anatomist to define all the boundary conditions between bones and soft tissues. Each rigid body is fixed to the nearby finite element nodes of the soft tissue mesh to naturally rigidify the foot. Each bone is automatically connected to its neighbor by a joint. In our model, those joints are simulated by 32 spherical pivots and one cylindrical pivot. The cylindrical pivot is between the talus and calcaneus bones and is placed below the talus (Figure 5). It constrains the motion of the calcaneus to a single rotation axis following the long axis of the foot. Spherical pivots have three rotation axes. The angles permitted by these pivots vary depending on their location to simulate the possible motions of actual foot joints, (Figure 6). For the phalanges, a maximum rotation angle of 45 degrees is allowed while the maximum allowable rotation of the metatarsal pivots is 30 degrees. The joints for the bones in the mid and back foot were limited to rotation angles between 0 and 5 degrees to simulate proper rigidity of the foot. The main motion of all the joints is around a rotation axis given by the intersection of the frontal and horizontal planes at the location of the corresponding joint.

\subsection{Ligaments}

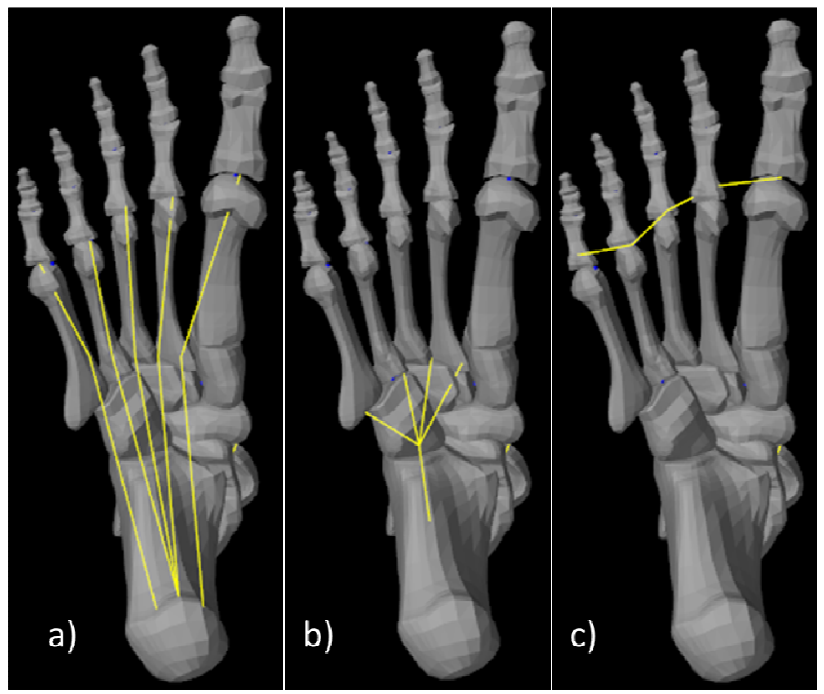

Figure 7. The main foot ligaments are simulated as cables. Posterior view of the models shows, a) the external plantar fascia, b) the internal plantar fascia, and c) the transversal metatarsal head ligament.

The foot is constrained by large ligaments to support body weight and to ensure stability. Our model integrates the six main ligaments of the foot. The plantar fascia connects the calcaneus to the proximal part of the first phalanx of each toe. In the model, it is separated in two sets: (1) the external fascia for the outer ligament layer (Figure 7a) and (2) the internal fascia for the inner ligaments close to the mid foot (Figure 7b). The transversal metatarsal head ligament connects the proximal part of the first phalanx of each toe to constrain lateral spreading of the fore foot (Figure 7c). The Achilles tendon connects the calcaneus to the calf and to the bottom part of the knee (Figures 8 and 9). In our model, the Achilles tendon is also separated into two sets: (1) the external tendon for the part closer to the surface and going towards the distal part of the calf, and (2) the internal tendon for the deeper part going towards the knee. Three smaller ligaments are also integrated in the model: the triangular ligament (Figure 8) between the navicular, calcaneus and cuboid bones, and two internal ligaments (Figure 9) between the calcaneus and navicular bones, and between the talus and navicular bones. 


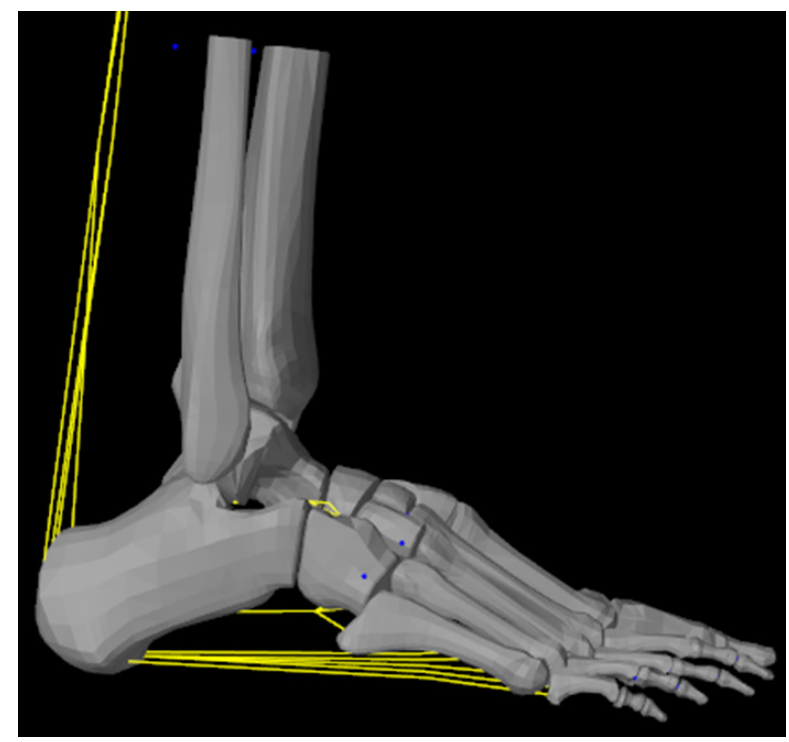

Figure 8. Lateral view of the main ligaments of our model: on the left, the Achilles tendon, and below the arch, horizontally, the two sets of plantar fascia. The triangular ligament linking the navicular, calcaneus and cuboid bones is visible near the ankle joint.

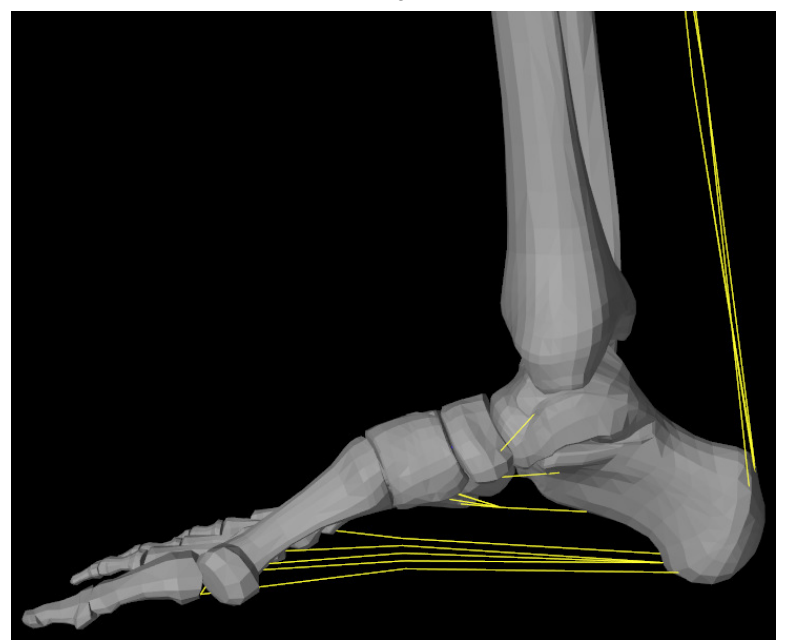

Figure 9. Medial view of the main ligaments of our model: on the right, the Achilles tendon, and below the arch, horizontally, the two sets of plantar fascia. The two internal ligaments linking the calcaneus and navicular bones, and the talus and navicular bones are visible near the ankle joint.

Ligaments are modeled as cables connecting the previously mentioned bony structures. Cables were chosen over anisotropic finite elements to reduce computation time, since this kind of elements would have required a much finer mesh to represent separate ligaments. Ligament attachments and paths have been manually created to guarantee precision of the bony insertion site for the ligaments. These insertion sites were defined by an experienced podiatrist. Based on the work of Gefen [21], we assigned different stiffness to the ligaments whether they are elongated or compressed. It has indeed been observed that they have a high stiffness when they are elongated, but no stiffness when they are compressed. Consequently, we assigned a stiffness value to the cables of $200 \mathrm{MPa}$ in extension and of $0 \mathrm{kPa}$ in compression. As a result, the ligaments resist elongation, but not compression.

\subsection{Boundary conditions}

As discussed in the introduction, internal tissues strains are likely the most significant factor for inducing deep pressure ulcers [22]. Therefore, it seems important to monitor these values, which is possible with our biomechanical model. To study the influence of a given foot position on internal strains, a set of pressures simulating the patient's foot in bipedal standing were applied to our biomechanical model. These plantar pressures were measured using a commercially available pressure sensor (Zebris platform, http://www.zebris.de/) under the right foot of a volunteer while standing. The left foot was also on the ground, but not on the pressure sensor, so that about half the body weight was applied onto each foot. The pressures ranged from 0 to $10.5 \mathrm{~N} . \mathrm{cm}^{-2}$ and are spread mainly below the heel and the metatarsal heads, Figure 10.

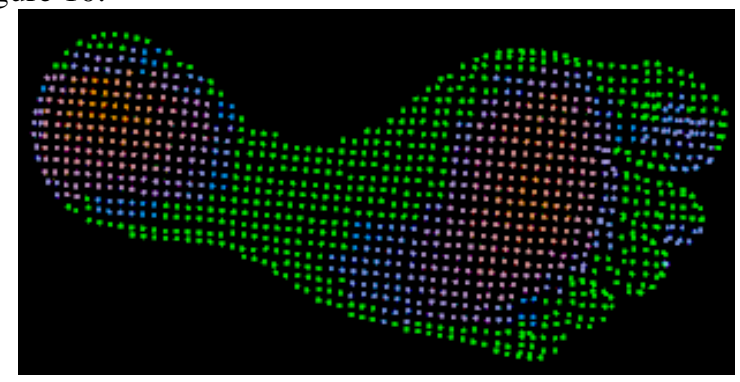

Figure 10. Distribution of the pressures applied under the right foot. Highest pressures, around $10.5 \mathrm{~N} . \mathrm{cm}^{-2}$, are in red (heel and metatarsal heads) and lower are in green.

The standing posture was simulated in two steps. First, the tibia and fibula bones were fixed and the rest of the foot was let loose under the influence of gravity for $0.2 \mathrm{~s}$ to reach a resting position. Secondly, from $t=0.2 \mathrm{~s}$ to $3 \mathrm{~s}$, the previously measured set of pressures was applied to the nodes of the foot sole following a slope $(0 \%$ at $0.2 \mathrm{~s}$ and $100 \%$ at 3 s) to model normal bipedal standing.

\section{Results}

Figures 11 and 12 show the Von Mises strains corresponding to the simulation at $\mathrm{t}=3 \mathrm{~s}$ at the time of maximum pressures applied to the sole of the foot. Table 1 summarizes the Von Mises strains at six key points of the foot: below the metatarsal head of each toe and below the heel, both at the skin surface and near the bones. The strains at the foot surface were markedly lower than the internal tissue strains. Maximal strains over the foot ranged from $2.7 \%$ to $8.0 \%$ for the skin surface and from $43.0 \%$ to $96.8 \%$ for the soft tissues near the bony structures. This strain distribution (with at least a 10 fold ratio between internal strains and foot surface strains) is consistent with 
the realistic behavior that is responsible for the creation of foot ulcers: they appear internally near the bones (because of very high strains) before being visible at the skin surface. Furthermore, the range of strains computed within the foot is consistent with the range of maximal strains observed in [13] for four subjects: between $52 \%$ and 107 $\%$.

Table 1. Von Mises (VM) strains measured under the metatarsal (MT) head of each toe and under the heel, internally (just below the bone structure) and externally (at the skin surface)

\begin{tabular}{|l|c|c|}
\hline Location & $\begin{array}{l}\text { Foot surface VM } \\
\text { strain }\end{array}$ & Internal VM strain \\
\hline $5^{\text {th }}$ toe MT & $2.7 \%$ & $63.3 \%$ \\
\hline $4^{\text {th }}$ toe MT & $5.2 \%$ & $96.8 \%$ \\
\hline $3^{\text {rd }}$ toe MT & $8.0 \%$ & $63.0 \%$ \\
\hline $2^{\text {nd }}$ toe MT & $4.1 \%$ & $84.2 \%$ \\
\hline $1^{\text {st }}$ toe MT & $5.1 \%$ & $43.0 \%$ \\
\hline Heel & $5.0 \%$ & $69.8 \%$ \\
\hline
\end{tabular}

Note that high strains are visible near the ankle in Figure 11. They result from the fact that the tibia and fibula are fixed while the rest of the bones are moving during the simulation, hence creating fairly large displacements near the ankle joints. During bipedal standing, the tibia and fibula would probably move slightly to adjust the position of the hips and the rest of the body, and consequently these high strains would probably be reduced. The sole of the foot is therefore the main region of interest in this simulation.

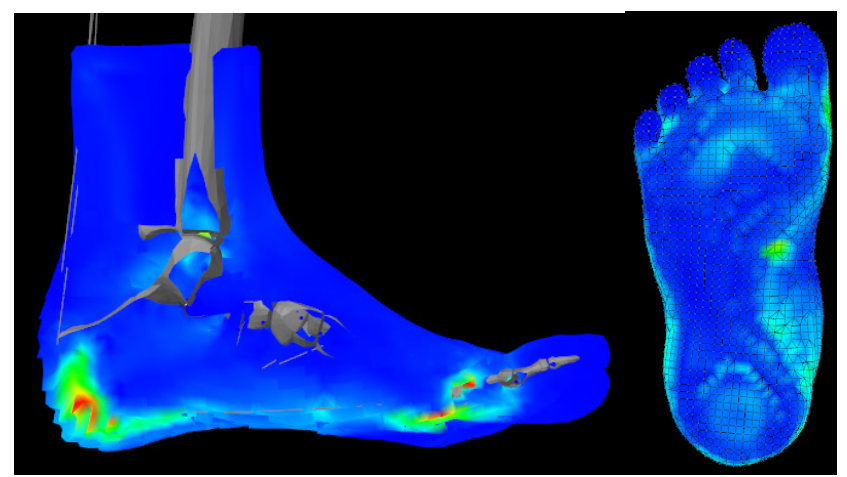

Figure 11. Left: cross section at the second toe showing the internal Von Mises strains resulting from the pressures applied to the foot sole and simulating a standing position. Right: Von Mises strains below the foot. The color map goes from no strain in blue to strains of $100 \%$ in red.

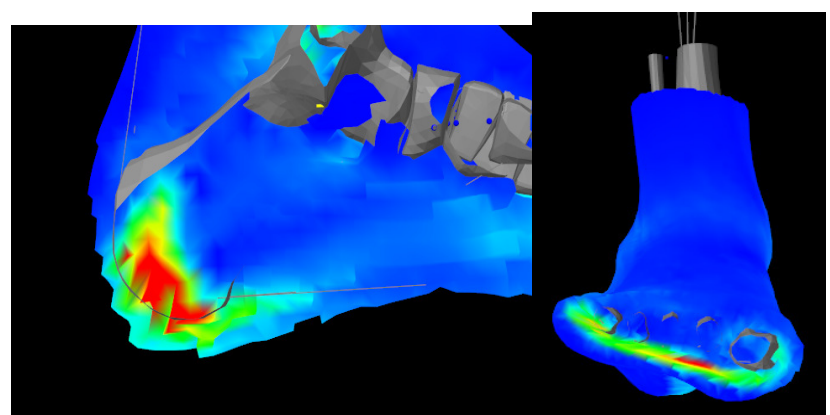

Figure 12. Left: cross section at the heel showing the internal Von Mises strains resulting from the pressures applied to the foot sole and simulating a standing position (the "staircase" shape of the foot surface is simply a visual artifact due to the clipping plane at the element boundary). Right: Von Mises strains at the metatarsal heads, front view. The color map goes from no strain in blue to strains of $100 \%$ in red.

The simulation takes 22 minutes to compute with ArtiSynth on a standard PC equipped with an Intel i5 2.80 $\mathrm{GHz}$ processor and 3.42 Go of RAM.

\section{Discussion and conclusion}

In this paper, we have proposed a biomechanical foot model to estimate the strains within the foot and determine whether ulcers may appear for given patient postures and plantar pressures. The goal of this work was to propose an anatomically accurate $3 \mathrm{D}$ foot model that can simulate at interactive rates.

Our foot model includes several structures. A FE mesh represents the foot soft tissues with four different Neo Hookean materials for plantar skin, non-plantar skin, the arch muscles and the fat in between those layers. Rigid bodies model the 26 foot bones that are connected with 33 pivots representing the joints. Three large ligaments (plantar fascia, transversal metatarsal head ligament, and Achilles tendon) and three smaller ligaments are also integrated in the model to connect certain bones and to stabilize the foot.

Simulations of foot loading with the model showed a realistic behavior in standing stance in terms of surface and internal strains with pressures measured with a commercial sensor. Average surface strains of $5 \%$ were evaluated below the heel and metatarsal heads while average strains of $70 \%$ were measured internally, next to the bones. These initial results are promising, and we are currently performing a thorough validation study.

A key interest of our 3D model is that different levels of strains were computed at different locations within the foot as shown in Table 1. This clearly demonstrates the advantage of a 3D model over a 2D model: the capability to study the variations of the internal strains in terms of the anatomy of the patient and the pressure pattern applied below the foot. Our analysis of the tissue strains resulting from a prescribed load at the sole of the foot reveals a localization of higher pressures inside the foot than on its surface. Consequently, the model could be coupled to the 
output of a pressure sensor to determine whether or not ulcers may appear as a prevention tool for diabetic patients.

Several limitations of our model need to be addressed before reaching this prevention goal. First, to be used by a given patient, the model needs to take into account the patient's morphology. It is obvious that the location of the bony structures and their shape is a key point in the process of creation of an ulcer. Therefore, using a patientspecific model would be the first step to achieve in order to claim ulcer prevention. Using the Mesh-Match-and-Repair (MMRep) algorithm [23][24], it is possible to generate patient-specific biomechanical models from an image dataset. From a complex dataset (CT scan or MRI), it gives extremely accurate results in terms of modeling. Nevertheless, these imaging modalities are rarely used during ulcer prevention. Using cheap and easily accessible image modalities, such as ultrasounds, X-rays, or even a Kinect scan of the skin [25], the result of the MMRep algorithm remains accurate to a precision of around 1.4 $\mathrm{mm}$ [25]. The algorithm computes a non-rigid transformation between the structures segmented in the medical dataset and the biomechanical model. This transformation is then applied to the original model to create a new model corresponding to the anatomy of the patient. Based on this process, it would be possible to build biomechanical model of the foot for each patient and to use it to quantify the internal pressures that could create ulcer for this specific case as could be used in a foot ulcer prevention. The LASTIC device could be used to characterize the patient's skin and help to make the foot model more patient specific. It takes about two minutes to measure the elastic modulus of the skin below the foot arch. Using that device for different locations on the foot could improve the elasticity values given to the model and therefore be closer to the mechanics of the patient's foot.

The second step towards reaching the goal of ulcer prevention is the daily monitoring of the patient's foot. Using a pressure sensor as heavy and as expensive as the Zebris platform, for instance, is only possible in a laboratory or a clinical setting. A lighter and less expensive pressure sensor would permit equipping every patient and monitoring their foot pressures on a daily basis. Using a similar technology than that recently employed for the conception of the TexiCare device dedicated to the prevention of seated buttock pressure ulcers for people with spinal cord injury [26], the "Smart Sock" sensor [27], also developed by Texisense (http://www.texisense.com/), combines both qualities since it is made of a $100 \%$ textile pressure sensing fabric wirelessly connected to a controller which can record and monitor the pressures all around the foot (not only under the sole). It can be used continuously during daily living activities. When combined to a biomechanical model such as ours, its measured pressures could be used to estimate the internal strains. If such strains overcome a threshold in duration or in intensity
[22], an alert would thus have to be sent to the patient to prevent ulcer formation.

Finally, a more accurate and timely monitoring of the internal tissue pressures will require a model that is capable of dynamic estimation of these pressures. The "Smart Sock" sensor would help in this direction since it would give continuous measurements of the external pressures. Nevertheless, moving from simulating static standing to dynamic motion is the third area that needs to be addressed for complete ulcer prevention. The main challenge will reside in adapting our model to reduce its computation time. Although our model is faster than most realistic foot $\mathrm{FE}$ models from the literature, in its current form, it is indeed too long to spend 22 minutes to compute internal stresses from a set of external pressures. For this reason, it is not currently possible to integrate this model in a real-time foot ulcer prevention device. Three possible solutions will be soon investigated to achieve real-time foot simulations: (1) decreasing the time of computation by reducing the number of elements in the areas less involved in ulcer formation (above the ankle for example), (2) precomputing accurate simulations with our current model and using them to evaluate the internal pressures from the continuously measured external pressures, or (3) limiting the FE modeling to a specific part of the foot such as the heel and/or the metatarsal heads. In any case, the foot model introduced in this paper will be useful since it now represents a reference in terms of modeling to which any "reduced" model will be compared.

\section{Acknowledgments}

This work is partly funded by the French national project ANR/TecSan 2010 IDS, the French national Investments for the Future program project VHP@interactive and by the CAMI Labex.

\section{References}

[1] J.E. Shaw and A.J.M. Boulton, "The pathogenesis of diabetic foot problems. An overview", Diabetes, 46 (Suppl 2), pp. S58-S61, 1997.

[2] V.R.. Driver, M. Fabbi, L.A. Lavery and G. Gibbons, "The costs of diabetic foot: the economic case for the limb salvage team", J Am Podiatr Med Assoc, 100(5), pp. 335$41,2010$.

[3] G.E. Reiber, "Epidemiology of Foot Ulcerations and Amputations in Diabetes", 6th ed., Mosby, 2010.

[4] M.J. Mueller, "Etiology, evaluation, and treatment of the neuropathic foot", Crit Rev Phys Rehabil Med, 3, pp. 289309, 1992.

[5] D.G. Armstrong and L.A. Lavery, "Evidence-based options for off-loading diabetic wounds", Clin Podiatr Med Surg, 15, pp. 95-104, 1998.

[6] A. Martinez-Nova, J.C. Cuevas-Garcia, J. Pascual-Huerta and R. Sinchez-Rodriguez, "BioFoot ${ }^{\circledR}$ in-shoe system: Normal values and assessment of the reliability and repeatability", The Foot, 17, pp. 190-196, 2007. 
[7] A.B. Putti, G.P. Arnold, L. Cochrane and R.J. Abboud, "The Pedar® in-shoe system: Repeatability and normal pressure values", Gait \& Posture, 25, pp. 401-405, 2007.

[8] A. Descatoire, A. Thévenon and P. Moretto, "Baropodometric information return device for foot unloading", Medical Engineering \& Physics, 31, pp. 607613,2009

[9] L. Pipkin and S. Sprigle "Effect of model design, cushion construction, and interface pressure mats on interface pressure and immersion", J Rehabil Res Dev, 45, pp. 875$882,2008$.

[10] E. Linder-Ganz, N. Shabshin, Y. Itzchak, Z. Yizhar, I. Siev-Ner and A. Gefen, "Strains and stresses in sub-dermal tissues of the buttocks are greater in paraplegics than in healthy during sitting", Journal of Biomechanics, 41, pp. 567-580, 2008.

[11] R. Sopher, J. Nixon, C. Gorecki, A. Gefen, "Exposure to internal muscle tissue loads under the ischial tuberosities during sitting is elevated at abnormally high or low body mass indices", Journal of Biomechanics, 43, pp. 280-286, 2010.

[12] J.J. Elsner and A. Gefen, "Is obesity a risk factor for deep tissue injury in patients with spinal cord injury?", Journal of Biomechanics, 41, pp. 3322-3331, 2008.

[13] G. Yarnitzky, Z. Yizhar and A. Gefen, "Real-time subjectspecific monitoring of internal deformations and stresses in the soft tissues of the foot: a new approach in gait analysis", J Biomech, 39 (14), pp. 2673-89, 2006.

[14] W.R. Ledoux, D.F. Meany and H.J. Hillstrom, "A quasilinear, viscoelastic, structural model of the plantar soft tissue with frequency sensitive damping properties", Journal of Biomechanical Engineering, 126, pp. 1-7, 2004.

[15] W.M. Chen, T. Lee, P. Vee-Sin Lee and S.J. Lee, "Effects of internal stress concentrations in plantar soft-tissue preliminary three-dimensional finite element analysis", Med. Eng. \& Phys., 32, pp. 324-331, 2010.

[16] J.E. Lloyd, I. Stavness and S. Fels, "ArtiSynth: a fast interactive biomechanical modeling toolkit combining multibody and finite element simulation", Soft Tissue Biomechanical Modeling for Computer Assisted Surgery, Studies in Mechanobiology, Tissue Engineering and Biomaterials, Vol.11. Springer, pp. 355-394, 2012.

[17] C. Lobos, Y. Payan and N. Hitschfeld, "Techniques for the generation of 3D Finite Element Meshes of human organs", Informatics in Oral Medicine: Advanced Techniques in Clinical and Diagnostic Tech. Hershey, PA: Medical Information Science Reference, pp. 126-158, 2010.

[18] R. Sopher, J. Nixon, E. McGinnis and A. Gefen, "The influence of foot posture, support stiffness, heel pad loading and tissue mechanical properties on biomechanical factors associated with a risk of heel ulceration", $J$ Mech Behav Biomed Mater, 4(4), pp. 572-82, 2011.
[19] I. Stavness, J. Lloyd, Y. Payan and S. Fels, "Coupled HardSoft Tissue Simulation with Contact and Constraints Applied to Jaw-Tongue-Hyoid Dynamics", International Journal for Numerical Methods in Biomedical Engineering, 27, pp. 367-390, 2011.

[20] V. Luboz, E. Promayon, G. Chagnon, T. Alonso, D. Favier, C. Barthod and Y. Payan, "Validation of a Light Aspiration device for in vivo Soft TIssue Characterization (LASTIC)", Soft Tissue Biomechanical Modeling for Computer Assisted Surgery, pp. 243-256, Springer Verlag, Berlin, 2012.

[21] A. Gefen, "Plantar soft tissue loading under the medial metatarsals in the standing diabetic foot", Medical Engineering \& Physics, 25, pp. 491-499, 2003.

[22] S. Loerakker, "The relative contributions of muscle deformation and ischaemia to pressure ulcer development", PhD TU Eindhoven, The Netherlands, 2011.

[23] M. Bucki, C. Lobos and Y. Payan, "A Fast and Robust Patient Specific Finite Element Mesh Registration Technique: Application to 60 Clinical Cases", Med. Im. An., 14, pp. 303-317, 2010.

[24] M. Bucki, C. Lobos, Y. Payan and N. Hitschfeld, "Jacobian-based Repair Method for Finite Element Meshes after Registration", Engineering with Computers, 27, pp. 285-297, 2011.

[25] M. Bucki, Y. Payan, F. Cannard, B. Diot and N. Vuillerme, "Multi-modal framework for subject-specific finite element model generation aimed at pressure ulcer prevention". Computer Methods in Biomechanics and Biomedical Engineering $(C M B B E)$, Proceedings of the Society of Biomechanics (SB) conference, September 2013.

[26] O. Chenu, N. Vuillerme, M. Bucki, B. Diot, F. Cannard and Y. Payan, "TexiCare: An innovative embedded device for pressure ulcer prevention. Preliminary results with a paraplegic volunteer", Journal of Tissue Viability, 22, pp. 83-90, 2013.

[27] M. Bucki, N. Vuillerme, F. Cannard, B. Diot, G. Becquet and Y. Payan, "The TexiSense « Smart Sock »- Textile Pressure Sensor and 3D Real-time Finite Element Model of the Diabetic Foot for a Daily Prevention of Pressure Ulcers". Proceedings of the 14th Annual European Pressure Ulcer Meeting, EPUAP, 2011. 